\title{
Obiettivi infermieristici nella gestione del catetere venoso centrale
}

\author{
E. Filardo, F. Marin \\ U.O. Nefrologia e Dialisi, Sanremo, ASL 1 Imperiese
}

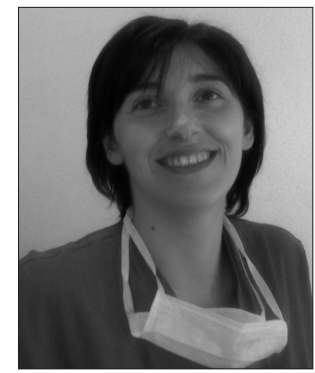

Ua gestione dei cateteri venosi centrali (CVC) è competenza dell'infermiere professionale che deve assicurare una corretta assistenza e prevenire le possibili complicazioni: ciò si ottiene tramite una uniformità e omogeneità di comportamento di tutto il personale utilizzatore. Nasce quindi la necessità di mettere a punto protocolli di gestione infermieristica del CVC, al fine di ottimizzare l'assistenza, sfruttare al massimo le potenzialità dello stesso e ridurre al minimo le complicanze. Un protocollo definisce $\mathrm{i}$ tempi, i modi, i materiali e il numero degli operatori dei quali avvalersi durante una qualsiasi procedura, per ottenere un sincronismo delle azioni, riducendo all'essenziale durata e risorse impiegate ed eliminando contemporaneamente il rischio di incorrere in dimenticanze che potrebbero favorire eventuali complicazioni.

Una buona comunicazione con l'Utente/Paziente, è infine, lo strumento fondamentale per erogare assistenza "di qualità".

In questa breve rassegna indicheremo quali sono gli obiettivi principali che perseguiamo nella nostra U.O. al riguardo della gestione routinaria infermieristica dei CVC.

\section{I cateteri venosi centrali}

I CVC sono utilizzati solitamente in ambiente ospedaliero, ma anche a domicilio dei pazienti, con la collaborazione dell'équipe medico-infermieristica in grado di indicare le idonee misure igienico sanitarie.

I CVC permettono di infondere per tempi più o meno prolungati liquidi di varia natura anche ad elevata velocità, di somministrare in modo controllato farmaci (ad esempio, terapie antiblastiche), nutrizione e soluzioni ipertoniche (che provocano flebiti se infuse in vene di piccolo calibro), di depurare l'organismo da tossine esogene o endogene (intossicazioni alimentari, farmacologiche, insufficienza renale acuta [IRA] e cronica [IRC]), di sottrarre liquidi in eccesso (pazienti con IRA, IRC, scompenso cardiaco), di monitorizzare la pressione venosa centrale ecc.

Non esistono controindicazioni assolute al posizionamento di un catetere venoso centrale, in quanto la puntura "ecoguidata" permette di incannulare vasi anche in pazienti che non hanno un adeguato assetto coagulativo e presentano alterazione di decorso dei vasi: unica controindicazione assoluta è la non visualizzazione ecografica di un vaso pervio. Le forme, i materiali, i siti di collocazione dei CVC sono diversi fra loro perché diverse sono le esigenze di ciascun paziente, ma tutti hanno un comune denominatore: la gestione è sempre affidata alla figura professionale dell'infermiere.

\section{Principali obiettivi della gestione del CVC}

Mantenimento del sistema chiuso. Permette da un lato di prevenire perdite ematiche e dall'altro di eliminare la contaminazione del "letto" vascolare. È ottenuto attraverso semplici manovre e costante attenzione da parte dell'Infermiere che lo gestisce. Infatti la chiusura del CVC è mantenuta da un lato dalle "clip" (una per lume nei CVC a due vie) presenti a livello del tratto "esterno" e dall'altro da tappini avvitati ai tratti terminali esterni. Dopo ogni utilizzo è necessario valutare sterilmente (guanti sterili e/o garza sterile imbibita di amuchina medicata o clorexidina al $2 \%$ ) con attenzione la corretta chiusura di tali presidi che, nel caso dei pazienti in emodialisi, devono restare a riposo per almeno 44 ore circa.

Mantenimento del sistema pervio (prevenzione delle trombosi endoluminali). Di importanza fondamentale per il buon funzionamento del CVC è la pervietà del/i lume/i che deve essere sempre verificata dall'infermiere prima di ogni utilizzo.

È ottenuto attraverso la rimozione di eventuale residui di sangue o soluzioni infusionali tramite un corretto lavaggio con soluzione fisiologica e l'utilizzo di un anticoagulante endoluminale. Infatti al termine dell'uso i lumi del CVC vanno sempre immediatamente lavati con soluzione fisiologica e riempiti con una idonea quantità (evidenziata solita- 
mente sui morsetti o sui lumi del CVC) di anticoagulante senza che quindi lo stesso sia infuso in circolo. Se i lumi non sono totalmente pieni di liquido di block, si verifica la formazione di coaguli endoluminali all'interno del CVC che, oltre ad occluderlo, potrebbe favorire lo sviluppo di colonie batteriche a volte particolarmente resistenti agli antibiotici. L'impiego del citrato sodico al 3.8\%, al posto della tradizionale eparina, ha notevolmente ridotto le complicanze: l'ambiente acido creato dal citrato blocca la sequenza della coagulazione nell'ultima fase, sequestrando gli ioni calcio indispensabili affinché la tromboplastina si trasformi in protrombina. Inoltre anche se il citrato passa in circolo è fisiologicamente metabolizzato in bicarbonato attraverso il ciclo dei citrati e pertanto un lieve eventuale sovraddosaggio non produce effetti anticoagulanti sistemici rilevanti (al contrario di quanto avviene con l'eparina). Il citrato sodico possiede inoltre un emivita più lunga dell'eparina, è più economico e contemporaneamente contrasta la crescita batterica attraverso l'abbassamento del $\mathrm{pH}$.

In caso di nutrizione parenterale è consigliabile destinare all'uso sempre la stessa via che sarà utilizzata e manutenuta in maniera costante, riservando la seconda a scorta o ad altri usi, in caso di problemi di flusso alla prima via: anche questa seconda via dovrà essere comunque lavata costantemente con sostituzione dell'anticoagulante.

Prevenzione nel danneggiamento del dispositivo. È effettuata utilizzando disinfettanti e detergenti idonei indicati dalla casa produttrice e compatibili con il materiale di cui è composto il CVC (nei CVC in poliuretano si è evidenziata una sensibilità per tutte le preparazioni con mupirocina e polietilenglicole, mentre per quelli in silicone sono banditi gli antisettici a base di iodio). In realtà, nella pratica clinica quotidiana, non si ha evidenza di danni importanti sulla struttura del CVC da parte delle differenti soluzioni disinfettanti/detergenti utilizzate ed è perciò probabile che questi ultimi non siano poi così dannosi. Non consigliabile comunque l'utilizzo di solventi, quali ad esempio, acetone ed etere, sul sito di inserzione o peggio sul CVC, durante il cambio della medicazione.

D'obbligo è inoltre l'abolizione dei taglienti nella manutenzione routinaria dei CVC onde prevenire eventuali danni.

Prevenzione delle infezioni. Il rischio di infezione nei pazienti portatori di CVC è influenzato da diversi fattori tra i quali le condizioni cliniche del paziente, il tipo di CVC, il tipo di infusione, il tipo di medicazione e la pulizia della cute. La prevenzione si ottiene attraverso un corretto utilizzo dei presidi di protezione individuale (PPI) (guanti sterili, camice idrorepellente, copricapo, occhiali paraspruzzi e mascherina chirurgica) e di materiale sterile. Nello specifico della gestione infermieristica dei CVC, il protocollo prevede di adottare manovre sterili: ideale è la cooperazione di due operatori, in quanto il primo esegue le manovre in sterilità, mentre il secondo lo assiste durante la procedura. In commercio esistono comunque kit sterili attacco/stacco che permettono ad un infermiere di lavorare in sterilità da solo. Entrambi gli operatori devono preventivamente eseguire il lavaggio antisettico delle mani, quindi indossare i PPI previsti dalla legge 626 per la sicurezza sul lavoro. Mentre gli occhiali paraspruzzi, il camice idrorepellente ed i guanti sono ad esclusivo vantaggio degli operatori, copricapo e mascherina offrono protezione anche al portatore di CVC: infatti quest'ultimo è da considerare un potenziale vettore di infezioni, potendo essere causa di autocontaminazioni. Una volta indossati i PPI è possibile per l'operatore non sterile procedere alla rimozione della medicazione: sollevare i bordi della medicazione da sostituire e rimuovere la medicazione permeabile, trazionandola parallelamente al piano cutaneo dal lato distale al sito di inserzione verso quello prossimale. Il CVC deve al contempo essere immobilizzato in prossimità del sito di inserzione per limitare i rischi di dislocazione.

L'operatore sterile ispeziona ora il sito di inserzione del CVC, controlla eventuali segni di flogosi, verifica la tenuta del sistema di fissaggio e deterge la cute con garza sterile imbevuta di amuchina medicata o clorexidina al $2 \%$ al fine di eseguire l'antisepsi del sito di inserzione e rimuovere eventuali residui di sangue coagulato.

Il secondo operatore collabora con il primo infermiere nella preparazione del campo sterile. Viene servito all'operatore sterile un set monouso preconfezionato fornito di telino sterile e almeno quattro garze sterili; inoltre sono necessarie per ogni lume una siringa sterile vuota della capacità di $5 / 10 \mathrm{~mL}$ per aspirare la soluzione di anticoagulante endoluminale e una siringa sterile della capacità di $20 \mathrm{~mL}$ con soluzione fisiologica per lavare immediatamente dopo il lume del CVC. Tali manovre sono eseguite dall'infermiere sterile che quindi posiziona sull'exite-site una garza imbibita di amuchina medicata all' $0.05 \%$. Al termine di queste manovre l'operatore sterile applica una medicazione «a piatto» con una garza sterile e con cerotto la fissa alla cute oppure una medicazione trasparente (ad esempio, Flexigrid pellicola con cerotto), che offre il duplice vantaggio di essere impermeabile e di permettere un costante controllo visivo dell'exite site e della cute circostante.

Durante l'utilizzo il CVC deve essere sempre protetto e avvolto in un telino sterile, riducendo al minimo le possibilità di contaminazione.

Al termine delle sopraindicate manovre, dopo aver effettuato quanto previsto per il mantenimento del sistema pervio, si chiude l'estremità del catetere con adattatori sterili e la si avvolge in una garza sterile fissandola alla cute con un cerotto a piatto. È una medicazione molto sicura perché blocca il CVC impedendogli ogni movimento ed eliminando il rischio di dislocazioni, particolarmente indicata nei cateteri non tunnellizzati o nei tunnellizzati appena inseriti.

Il cerotto usato può lasciare però sia sulla cute del portatore che sul catetere residui considerevoli di colla che, oltre a creare infiammazione cutanea, causano prurito e conseguenti lesioni da grattamento con possibile contaminazione di patogeni. Una soluzione soddisfacente consiste nell'avvolgere il CVC in una garza sterile e chiuderla alla base con cerotto, introducendolo 
quindi in un sacchetto di garza sterile preconfezionato che viene fissato alla cute sempre tramite un cerotto. Il sacchetto dovrebbe essere sostituito tutte le volte che il CVC viene utilizzato, ma è utilizzabile per un massimo di sette giorni se integro e in condizioni igieniche buone. Questo presidio è solitamente ben tollerato da pazienti ed è sicuro come una "medicazione a piatto". La medicazione del CVC da emodialisi andrebbe cambiata ogni 48 ore per le medicazioni effettuate con garza, ogni 7 giorni per le medicazioni trasparenti e comunque ogni qualvolta il CVC venga utilizzato, o si osservino arrossamenti e/o secrezioni, o la medicazione risulti manomessa.

A ogni medicazione è necessario un meticoloso controllo dell'exite site mediante ispezione visiva e palpazione, al fine di verificare che non siano presenti segni di flogosi (gonfiore, rossore) e/o d'infezione (secrezioni sierose o purulente), trazioni sulla cute o sul catetere, anomalie meccaniche del catetere (angolature, rotture, perdite) e che sia garantita la tenuta dei punti di sutura (per i CVC temporanei o per i definitivi appena posizionati) e la corretta posizione dello stesso.

In caso di sospetta infezione locale del sito di inserzione (presenza di secrezioni), deve sempre essere eseguito un tampone colturale: pomate antibiotiche $o$ antisettiche non devono essere utilizzate sul sito di inserzione perché possono favorire infezioni fungine e resistenze antimicrobiche.

In caso di insorgenza di iperpiressia in un paziente portatore di CVC, al fine di porre eventuale diagnosi di batteriemia CVC correlata, occorre procedere all'esecuzione di emocoltura periferica controlaterale e direttamente da entrambi i lumi del CVC per il timing di crescita.

È chiaro che la partecipazione del paziente alla corretta gestione del CVC è di fondamentale importanza nella prevenzione delle infezioni: il paziente deve perciò essere invitato a riferire prontamente qualsiasi sintomo o situazione anomala. 